\title{
EVALUACIÓN DE DOS ADYUVANTES EN LA FORMULACIÓN DE UNA VACUNA ANTITETÁNICA
}

\author{
EVALUATION OF TWO ADJUVANTS FOR THE \\ FORMULATION OF ANTITETANIC VACCINES
}

\author{
Janeth del C. Arias ${ }^{1}$ \\ Diana C. Sánchez ${ }^{1}$ \\ Mery C. Vásquez C. ${ }^{1}$ \\ Cindy M. Fernández ${ }^{1}$ \\ Luz Marina Parra ${ }^{2}$
}

\section{RESUMEN}

El objetivo de esta investigación fue el de comparar el comportamiento de dos adyuvantes, hidróxido de aluminio y fosfato de calcio, con dos lotes diferentes de toxoide tetánico, uno producido por cultivo agitado y el otro producido por cultivo estático. Fue evaluada la respuesta inmune generada a través de la prueba de potencia del National Institute of Health $(\mathrm{NIH})$ para garantizar el cumplimiento de los estándares establecidos para la prueba de potencia, en la cual se postula que la respuesta inmune generada debe ser como mínimo de $2 \mathrm{UI} / \mathrm{mL}$. Los resultados muestran que el aluminio presenta títulos más altos en la mayoría de los casos y en otros no se aprecia una diferencia significativa entre los dos adyuvantes. También se observó claramente que cualquiera de los dos adyuvantes genera una mejor respuesta inmune con el lote 69 (cultivo estático) que con el lote 11 (cultivo agitado). El análisis de varianza arroja un error de $\mathrm{p}<0,002$.

${ }^{1}$ Grupo Biotecnología Ambiental e Industrial. Departamento de Microbiología. Facultad de Ciencias. Pontificia Universidad Javeriana, Dirección para Correspondencia Carrera 7 No. 43-82 Edificio Félix Restrepo. Bogotá-Colombia. Correspondiente autor: jdcarias@javeriana.edu.co, cinfi07@yahoo.com.

2 Instituto Nacional de Salud. Subdirección Industrial. Laboratorio de Tétanos. imparra@ins.gov.co.
Palabras clave: Tétano, adyuvante, fosfato de calcio, hidróxido de aluminio, límite floculante

\section{SUMMARY}

The objective of this research was to compare the role of two adjuvants, aluminum hydroxide and calcium phosphate, with two different tetanus toxoid batches, one obtained by stationary culture and the other one by sumerged culture. The generated immune response was evaluated through the potency assay of the National Institute of Health $(\mathrm{NIH})$, which states that in order to guaranty that the vaccines are within the established standards the immune response has to be at least $2 \mathrm{UI} / \mathrm{mL}$. The results indicate that aluminum hydroxide presents in most cases better titers, while in other cases no significant difference was found. Results showed also, that both adjuvants gave a better immune response with batch 69 (static culture) than with batch 11 (sumerged culture). The variance analysis showed an error of $\mathrm{p}<0.002$.

Key words: Tetanus, adjuvant, calcium phosphate, aluminum hydroxide, flocculent limit.

\section{INTRODUCCIÓN}

El hidróxido de aluminio, el aluminio fosfato y el fosfato de calcio han sido hasta el momento los adyuvantes 
aceptados por la Farmacopea Europea para vacunas humanas. Sin embargo, los adyuvantes que contienen aluminio están siendo reevaluados, ya que se ha demostrado la existencia de efectos adversos para los pacientes (Gupta \& Relyveld, 1991). La discusión sobre estas reacciones indica que el aluminio ha sido asociado a dolores, nódulos subcutáneos, formación de granulomas e inclusive desórdenes del sistema nervioso, como demencia de Altzheimer y sclerosis lateral amyotrófica. Todo esto se debe a la acumulación lenta de residuos de aluminio. Se plantea entonces que, como el Ca3(PO4)2, es un constituyente normal del cuerpo humano, probablemente produzca menos o ninguna reacción adversa.

Entre 1986 y 1991, se registraron 1.800 .000 episodios de tétanos al nivel mundial, de los cuales 1.200 .000 resultaron mortales, poniendo de relieve la importancia de su tratamiento y su erradicación. El tratamiento, se realiza por inmunización tanto pasiva como activa. En Colombia, el Instituto Nacional de Salud (INS, 1998), estaba encargado de la producción de la vacuna tetánica en sus tres presentaciones (Td, DPT y TT), siguiendo los lineamientos de la Organización Mundial de la Salud (OMS) (Barreto, 2000). El INS obtenía el toxoide tetánico a partir de cultivos sumergidos estacionarios de Clostridium. tetani en jarros de vidrio de 60L, utilizando el medio y los procedimientos sugeridos por Latham et al. (1962).

La enfermedad tiene distribución mundial, pero su foco es en áreas tropicales y en países en vías de desarrollo. Las características ecológicas propicias para el bacilo tetánico y las condiciones de higiene y sanidad favorecen que se presente con mayor frecuencia en las zonas rurales que en las urbanas. A pesar de ser una enfermedad previsible por vacunación, en los países en vías de desarrollo, entre ellos Colombia, muchas madres, no inmunizadas, dan a luz niños a quienes no se cuida en forma apropiada el cordón umbilical, por lo cual, el tétanos neonatal tiene un impacto significativo.

Aunque, se han publicado reportes de estudios de producción de toxina tetánica en fermentadores agitados a escala industrial, la carencia de instalaciones condujo a que el INS mantuviera la tecnología de cultivos estáticos. Aunque la tecnología de cultivo estático es sencilla, implica considerable manipulación, altos riesgos de contaminación tanto para el operario como para el cultivo, costos elevados, aumento de factores que conllevan a variaciones entre lote y lote y la dificultad de escalamiento de la producción (U.S. Health Human Services, 2003). Adicionalmente, la producción no alcanza a cubrir las necesidades de la población colombiana, obligando a importar una parte de la vacuna necesaria en los procesos de inmunización preventiva al nivel nacional. Por todas estas razones, el INS detuvo la producción de la vacuna, dejando vía libre solo para la importación. Sin embargo, por la bien conocida crisis nacional, no se ha importado lo suficiente para la demanda, lo que hoy nos coloca en emergencia sanitaria. De aquí la urgencia y la importancia de reactivar la producción de la vacuna.

Si bien el 80\% de los niños de todo el mundo en 1994 estaban vacunados contra el tétanos (con la vacuna DPT) durante su primer año de vida, más del $50 \%$ de los lactantes nacían sin protección contra la enfermedad porque sus madres no estaban vacunadas con el toxoide tetánico (OMS, 1997).

Definición y función de los adyuvantes: Un adyuvante inmunógeno puede ser definido como cualquier sustancia que incorporada a una vacuna, acelera, prolonga o potencia la respuesta inmunogénica frente a la misma. Son sustancias o preparados químicos que se incorporan al antígeno o inyectados simultáneamente con él y que hacen más efectiva la respuesta inmune. Con su empleo, se logra una economía de antígeno y de tiempo, así como un mayor nivel de anticuerpos específicos (Roitt, 1991).

Tienen gran importancia y se diferencian de las proteínas transportadoras o "carriers" (proteínas extrañas que se unen a un antígeno no inmunógeno para convertirlo en inmunógeno), en que no forman uniones estables con el inmunógeno. Los adyuvantes son básicamente necesarios en la inmunización inicial, mientras que los portadores, se necesitan tanto en la respuesta primaria como en las sucesivas. La mayoría de las nuevas vacunas compuestas por subunidades antigénicas altamente purificadas son muy seguras pero de menor inmunogenicidad que otras vacunas con más impurezas. Esta inmunogenicidad puede ser potenciada por los adyuvantes. La utilización de estos compuestos tiene como ventajas: a) inmunización eficaz de personas con capacidad inmune disminuida, como neonatos, ancianos y personas inmunodeprimidas; b) elaboración de 
vacunas con menos cantidad de antígeno y, por tanto, más aptas para crear vacunas combinadas y, c) menor número de dosis de refuerzo (Roitt, 1991).

El mecanismo de acción de estas sustancias ha sido objeto de numerosos estudios y, al parecer, existen diversos factores que explican su modo de acción. El antígeno libre normalmente se difunde con mucha rapidez desde los tejidos locales que rodean el sitio de inoculación, y una de sus funciones importantes es crear un reservorio o depósito del antígeno de larga vida. Las investigaciones realizadas han demostrado que virtualmente todos los adyuvantes activan o estimulan los macrófagos (Guerrero \& Gattas, 1982); cuando son activados estimulan la respuesta inmune por un incremento de la cantidad de antígeno expresado en la membrana celular y de la eficiencia de su presentación a los linfocitos. El macrófago también libera factores solubles estimulantes que amplifican la proliferación de los linfocitos (Wagnerová \& Ferencik, 1993). Por otro lado, algunos adyuvantes poseen la capacidad de actuar específicamente sobre los linfocitos, pero, en general, éstas funcionan mejor si facilitan la liberación simultánea del antígeno y de sustancias inmunomoduladoras al tejido linfoide (Bennet et al. 1992).

Tipos de adyuvantes: La búsqueda de nuevas sustancias con actividad adyuvante/inmunopotenciadora comprende una de las tendencias más importantes en la investigación inmunológica actual. Las sustancias inmunomoduladoras constituyen una familia muy heterogénea si se toma en consideración su origen, su naturaleza química y su actividad biológica específica (Takx-Kohlen, 1992). Los agentes inmunopotenciadores (adyuvantes) son la directriz del esfuerzo investigativo sostenido para hallar sustancias con actividad de adyuvantes, eficaces en la estimulación de la respuesta inmune y desprovistas de propiedades biológicas adversas (Stewart-Tull, 1989). Son importantes, ya que contribuyen a que las respuestas en animales de experimentación sean mayores, especialmente en aquellos casos en los que son empleados durante la producción de sueros hiperinmunes (Azuma, 1992).

De acuerdo a Guerrero \& Gattas (1982), entre los adyuvantes más importantes figuran:

- Alúmina: sales insolubles de sulfato alumínico-potásico y actúa mediante varios mecanismos, tales como
1.- Precipita el antígeno. Al inyectarse va liberando el antígeno lentamente, con lo que se suministra un estímulo persistente (el Ag dura varios días en el lugar donde se inoculó). 2.- El Ag precipitado tiene mayor tamaño, por lo que puede ser fagocitado más fácilmente y, por lo tanto, es presentado más efectivamente, y 3.- Inducción de granulomas.

- Adyuvantes de Freund: el adyuvante incompleto de Freund consiste en una solución acuosa con el Ag, junto con aceite mineral y un agente dispersante (el manoleato). El adyuvante completo de Freund (FCA) incorpora además una suspensión de Mycobacterium muertos por calor. Ambos liberan lentamente el Ag, logrando un estímulo persistente. El macrófago aumenta en su superficie el número de moléculas B7, lo que facilita su interacción con el receptor CD28 del linfocito $T_{H}$. Esto suministra la llamada señal coestimulatoria, que potencia la interacción entre MHC (del macrófago), Ag procesado y TCR (receptor de la célula T). Además, el completo es más potente porque suministra muramil-dipéptidos de la pared celular de las micobacterias, ello permite una buena activación de macrófagos, que liberan la citoquina IL-1, que a su vez activa a los linfocitos $\mathrm{T}_{\mathrm{H}}$. El completo induce mejor los granulomas que corresponden una infiltración celular, con una masa densa y rica en macrófagos, con lo que se mejora el procesamiento y la presentación del Ag. Se provoca una buena liberación de IL-1 de los macrófagos, que activan a los linfocitos $T_{H}$ (Guerrero $E$ Gatta, 1982).

- Polirribonucleótidos sintéticos: estimulan la proliferación inespecífica de linfocitos (Guerrero $\mathcal{E}$ Gattas, 1982)

- Lipopolisacárido bacteriano (LPS): igual efecto que el anterior. Recientemente se están ensayando los liposomas: el antígeno se encierra en liposomas o se une a la bicapa lipídica de este tipo de vesículas membranosas (Roitt, 1991)

- Compuestos de Aluminio: En la actualidad, los únicos adyuvantes que se utilizan son los compuestos de aluminio (hidróxido de aluminio y fosfato de aluminio). El ión aluminio genera exclusivamente respuestas de linfocitos $T$ ayudadores $\left(\mathrm{T}_{\mathrm{H}} 2\right)$, siendo muy útiles cuando se quieren obtener títulos de anticuerpos muy 
elevados, que neutralicen toxinas (los toxoides tetánico y diftérico) o virus, antes de que se adhieran al receptor celular (vacuna de hepatitis) (Roitt, 1991).

- Otros Adyuvantes: Existen otros adyuvantes con propiedades muy interesantes, algunos de los cuales están en fase de evaluación, como componentes bacterianos naturales o sintéticos (lípido A de las endotoxinas bacterianas y dipéptidos de muramilo); adyuvantes particulados, (complejos inmunoestimulantes, liposomas y bioesferas degradables); emulsiones oleosas, (saponinas y escualenos) y otros adyuvantes sintéticos, como los copolímeros de bloqueo no-iónico (Spriggs \& Koff, 1991).

Mecanismos de acción de los adyuvantes: Los adyuvantes, en general, actúan mediante tres mecanismos (Takx-Kholen, 1992):

- "Efecto depot", por el que los adyuvantes de aluminio, las emulsiones oleosas y los liposomas o microesferas mantienen al antígeno atrapado en el sitio de la administración de la vacuna, permitiendo un estímulo inmune prolongado. Este efecto es particularmente importante cuando se utilizan pequeños antígenos solubles que podrían ser aclarados rápidamente. Además, el mismo "efecto depot" impide una liberación masiva del antígeno, que podría dar lugar a tolerancia inmune, y causar una respuesta inflamatoria local que atrae a macrófagos y a otras células presentadoras de antígenos.

- Mejorar la presentación del antígeno a las células APC (Células Presentadoras de Antígenos) y actuando como coestimuladores de los macrófagos, como en el caso de los iones de aluminio, liposomas y copolímeros de bloqueo no-iónico.

- Induciendo la secreción de citocinas que actúan sobre los linfocitos T y B.

Adyuvantes como los péptidos de muramilo vehiculizados en aceite, el lípido A de las endotoxinas y el adyuvante completo de Freund estimulan preferentemente respuestas $T_{H} 1$, que dan lugar a citotoxicidad. En el mismo sentido, las saponinas, los complejos estimulantes inmunes y los liposomas, desencadenan respuestas citotóxicas aptas para destruir virus u otros organismos intracelulares (Takx-Kohlen, 1992).
Los adyuvantes derivados de las bacterias, como el lípido A y los péptidos de muramilo de la pared celular son muy potentes. Se ha postulado que sería debido a su reconocimiento por receptores de las células presentadoras de antígeno, cuya función es reconocer estructuras de bacterias, que se hayan conservado a lo largo de la evolución filogenética (Takx-Kohlen, 1992).

Algunas citocinas podrían ser utilizadas como adyuvantes, dada su capacidad para favorecer respuestas $\mathrm{T}_{\mathrm{H}} 1 \mathrm{o}$ $\mathrm{T}_{\mathrm{H}} 2$. Han sido utilizado para lograr respuestas a la vacuna de hepatitis $\mathrm{B}$ en personas que no desarrollan anticuerpos frente a la misma. Sin embargo, el uso de citocinas, como adyuvantes, se puede ver dificultado por las numerosas e imprevistas acciones de estos mediadores en el sistema inmune. La elección de un adyuvante determinado en la elaboración de una vacuna, depende de la naturaleza de ésta, del efecto deseado y de esto dependerá la efectividad de la vacuna (Takx-Kohlen, 1992).

Futuro de los adyuvantes: Las propiedades inmunomoduladoras han sido demostradas en numerosos polisacáridos naturales y algunos derivados obtenidos por hidrólisis o modificación química. La actividad estimulante de la hematopoyesis y de la respuesta inmune humoral y celular es uno de los rasgos más significativos de los glucanos aislados de la pared celular de levaduras (Diluzio, 1985). Ha sido mostrado que la administración simultánea del glucano y un antígeno estimula la formación de anticuerpos específicos contra el antígeno en cuestión; prueba de ello es la estimulación por los glucanos de la respuesta humoral y mediada por células contra Francisella tularensis y Pseudomonas pseudomallei (Reynolds et al. 1980).

El objetivo de esta investigación fue comparar el comportamiento de los dos adyuvantes, hidróxido de aluminio y fosfato de calcio utilizando dos lotes diferentes de toxoide tetánico, uno producido por cultivo agitado y el otro producido por cultivo estático. La comparación fue evaluada mediante la respuesta inmune generada a través de la prueba de potencia del National Institute of Health $(\mathrm{NIH})$, de tal forma que garantice el cumplimiento de los estándares establecidos para la prueba de potencia, donde se postula que la respuesta inmune generada debe ser mínimo de $2 \mathrm{UI} / \mathrm{mL}$. También se buscó el mejor adyuvante frente a la respuesta inmune generada y la relación existente entre el método de producción y la respuesta inmune. 


\section{MATERIALES Y MÉTODOS}

Vacunas: Doce vacunas, todas diferentes, fueron preparadas de acuerdo a las Buenas Prácticas de Manufactura (BPM). Para la preparación de estas vacunas, se utilizó un lote de toxina producido por cultivo agitado (11) y un lote de toxina producido por cultivo estático (69) facilitados por el Instituto Nacional de Salud (INS). De cada lote, se formularon seis vacunas, de las cuales tres se adsorbieron en $\mathrm{Al}(\mathrm{OH})_{3}$, en una concentración por vacuna $(<1,25 \mathrm{mg} / \mathrm{mL})$ y las otras tres restantes se adsorbieron en $\mathrm{Ca}_{3}\left(\mathrm{PO}_{4}\right)_{2}$, en una concentración por vacuna $(<1,3 \mathrm{mg} / \mathrm{mL})$. Cada vacuna llevó como agente conservador Timerosal al $1 \%$ en una concentración por vacuna $(<0,022 \%)$ y se diluyó en solución salina tamponada $\mathrm{pH} 7,2$. Cada una de las doce vacunas, se sometieron a las pruebas de control de calidad exigidas por el registro de producción de vacuna antitetánica adsorbida del INS (INS, 1995).

Prueba de Potencia: Se utilizó el método de potencia del National Institute of Health (NIH), donde la inmunización se hace en cobayos cepa Hartley y el desafío se realiza con ratones cepa $\mathrm{NIH}$. El análisis de la potencia se realiza por el método estadístico de Spearman $\mathcal{E}$ Karber. Para cada una de las doce vacunas, se realizó la prueba de potencia por triplicado, para hacer el estudio estadísticamente representativo.

Inmunización: Para la inmunización, se utilizaron 18 cobayos Cepa Hartley con un peso entre 350 - 450g por cada vacuna, cada uno con la mitad de la dosis humana inmunizante $(1,5 \mathrm{~mL})$ por vía intraperitoneal. La inmunización dura 45 días durante los cuales se pesan los animales semanalmente para verificar que no presentan efectos adversos y que suban de peso.

Sangría: Se anestesia cada cobayo con Ketamina $(50 \mathrm{mg} / \mathrm{mL})$ y Seton (20mg Xilacina/mL). Posteriormente, se colectó por punción cardíaca $10 \mathrm{~mL}$ de sangre de cada animal. Los tubos se incubaron a $4^{\circ} \mathrm{C}$ por $24 \mathrm{~h}$. Se centrifugaron a 4000 r.p.m. por 15 minutos y se extrajo el suero con pipeta en tubos secos, luego, se midió el volumen de suero del tubo que contenía menos cantidad del mismo y con este volumen se realizó un pool de suero.

Titulación del Suero: Se realizaron cuatro diluciones a una actividad supuesta de 2, 4, 8 y 16UI, con un factor de dilución de dos para cada muestra. Para el control de la prueba, se utilizó una antitoxina de referencia obtenida del Instituto Butantan, Sao Paulo, Brasil, titulada a 1 UI, con la cual se realizó cuatro diluciones a un titulo supuesto de 0,$69 ; 0,83 ; 1,0$ y 1,20 UI, con un factor de dilución de 1,2.

A cada tubo, tanto de control como de muestra, se le adicionó un volumen constante de toxina tetánica patrón (Butantan), titulada a 1UI, de tal manera que el volumen inoculado por animal contenía una dosis $L+/ 10$. Todos los tubos se agitaron e incubaron a $37^{\circ} \mathrm{C}$ por 45 minutos e inmediatamente se inocula $0,5 \mathrm{~mL}$ de la mezcla por vía subcutánea, a seis ratones, cepa $\mathrm{NIH}$, por tubo de mezcla. Los animales, se observaron diariamente por un período de 96 horas y se registró la mortalidad. Los resultados, se procesaron por el método estadístico de Spearman $\mathcal{E}$ Karber, para informar la potencia en $\mathrm{UI} / \mathrm{mL}$.

\section{RESULTADOS Y DISCUSIÓN}

La prueba de potencia, se realizó en tres pasos básicos: Inmunización, Sangría y Titulación de sueros, según el Procedimiento Operativo Estándar del Laboratorio de Tétanos del INS (1995). Los resultados de la actividad inmunogénica, para cada vacuna, se observan en la Tabla 1. Se evidencia que el aluminio presenta títulos más altos en algunos casos y en otros no se aprecia una diferencia significativa. También, se nota claramente que cualquiera de los dos adyuvantes genera una mejor respuesta inmune con el lote 69 (cultivo estático) que con el lote 11 (cultivo agitado).

El análisis estadístico, se realizó con la prueba de Scheffe, que permitió identificar cuál nivel del adyuvante de cada ensayo hace rechazar la hipótesis de trabajo, el ANOVA arrojó un error de $(\mathrm{p}<0,002)$. En la Tabla 2 , al observar los números de Scheffe, se evidencia que no existe una gran diferencia entre los dos adyuvantes, por ende se rechaza la hipótesis nula y determinar que para esta variable cada uno de los adyuvantes tiene un comportamiento diferente en cuanto a la variable respuesta (respuesta inmunogénica) y no tiene ninguna similitud en los datos.

En la Gráfica 1 se muestra el comportamiento de los dos adyuvantes frente a la respuesta inmunogénica, 
Tabla 1: Resultados de la prueba de potencia de cada vacuna.

\begin{tabular}{|c|c|c|c|c|c|}
\hline LOTE & Adyuvante & Vacuna 1 & Vacuna 2 & Vacuna 3 & $\begin{array}{l}\text { Antitoxina de } \\
\text { Referencia }\end{array}$ \\
\hline \multirow{6}{*}{11} & & $1 \mathrm{~A}$ & 2,8284 UI & اU 5,6569 & 1,0627 \\
\hline & $\mathrm{Al}(\mathrm{OH})_{3}$ & $1,4142 \mathrm{UI}$ & 2,2449 UI & $5,0396 \mathrm{UI}$ & 1,0627 \\
\hline & & 1,5874 UI & 2,8284 UI & ال & 1,0627 \\
\hline & & $1,4142 \mathrm{UI}$ & 2,8284 Ul & $5,0397 \mathrm{UI}$ & 1,0627 \\
\hline & $\mathrm{Ca}_{3}\left(\mathrm{PO}_{4}\right)_{2}$ & 1,4142 UI & 1,7817 & 4,4898 Ul & 1,0627 \\
\hline & & 1,7817 UI & 2,2449 UI & $4,0000 \mathrm{UI}$ & 1,0627 \\
\hline \multirow{6}{*}{69} & & $7,1272 \mathrm{UI}$ & 11,3137 UI & $13,3333 \mathrm{UI}$ & 1,0627 \\
\hline & $\mathrm{Al}(\mathrm{OH})_{3}$ & 7,1271 UI & 11,3137 U & 12,6962 UI & 1,0627 \\
\hline & & 6,3496 UI & I1,3137 I & 12,6992 UI & 1,0627 \\
\hline & & 6,3496 I I & $8,0000 \mathrm{UI}$ & I1.3137 U & 1,0627 \\
\hline & $\mathrm{Ca}_{3}\left(\mathrm{PO}_{4}\right)_{2}$ & ال & 7,1272 Ul & $12,6992 \mathrm{UI}$ & 1,0627 \\
\hline & & $10 \mathrm{C}$ & $11 C$ & $12 C$ & \\
\hline
\end{tabular}

Tabla 2. Prueba de rango múltiple. Variable adyuvante.

\begin{tabular}{|l|l|l|l|}
\hline \multicolumn{4}{|c|}{ MÉTODO: 95,0\% LSD } \\
\hline ADYUVANTE & \# ENSAYOS & Promedio Scheffe & Grupos Homogéneos \\
\hline Hidróxido Al (1) & 18 & 6,77487 & $\mathrm{X}$ \\
\hline Fosfato Ca (2) & 15 & 5,65096 & $\mathrm{X}$ \\
\hline & CONTRASTE & DIFERENCIA & $+/$ - LIMITES \\
\hline & $1-2$ & $* 1,12391$ & 0,314628 \\
\hline
\end{tabular}

* Diferencia estadísticamente significativa.

independientemente de los lotes. Claramente se observa la mejor efectividad del hidróxido de aluminio. En cuanto a la interacción entre los dos adyuvantes con respecto a los dos lotes de producción, en la Gráfica 2, se observa que ambos adyuvantes con el lote 69 presentan mejores resultados que con el lote 11 .
La comparación entre el comportamiento de la variable adyuvante frente a la respuesta inmunogénica determina que el hidróxido de aluminio es mejor que el fosfato de calcio. Según Aggerbeck et al. (1995), el adyuvante hidróxido de aluminio se une fuertemente al antígeno que el fosfato de calcio, esto implica que esa unión tan 


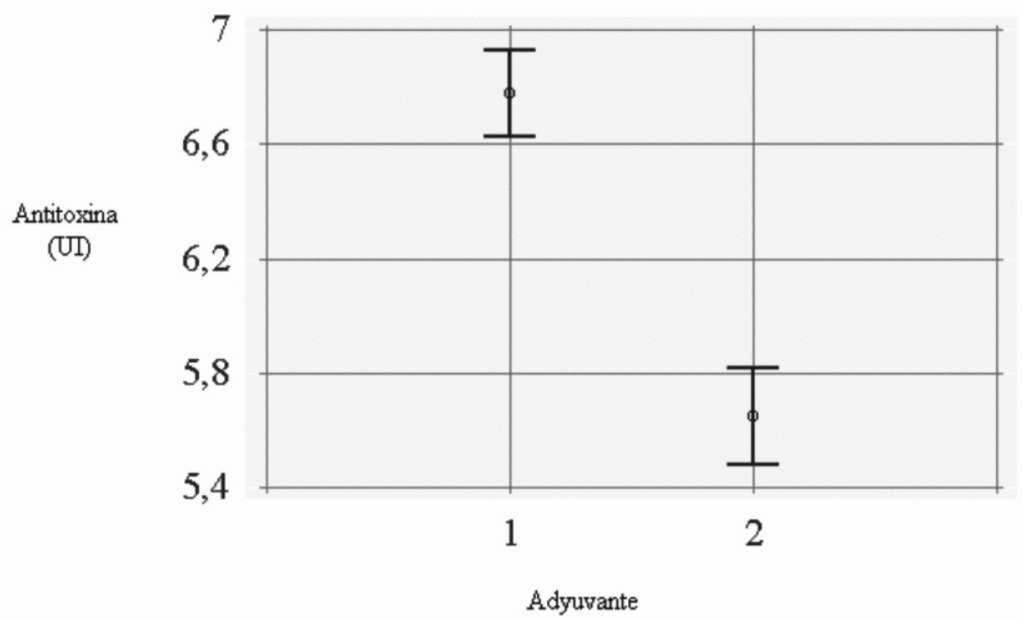

Gráfica 1. Comparación estadística entre los adyuvantes con respecto a la respuesta inmunogénica.

\section{INTERACCION ENTRE EL METODO DE PRODUCCION DE LOS LOTES Y EL ADYUVANTE}

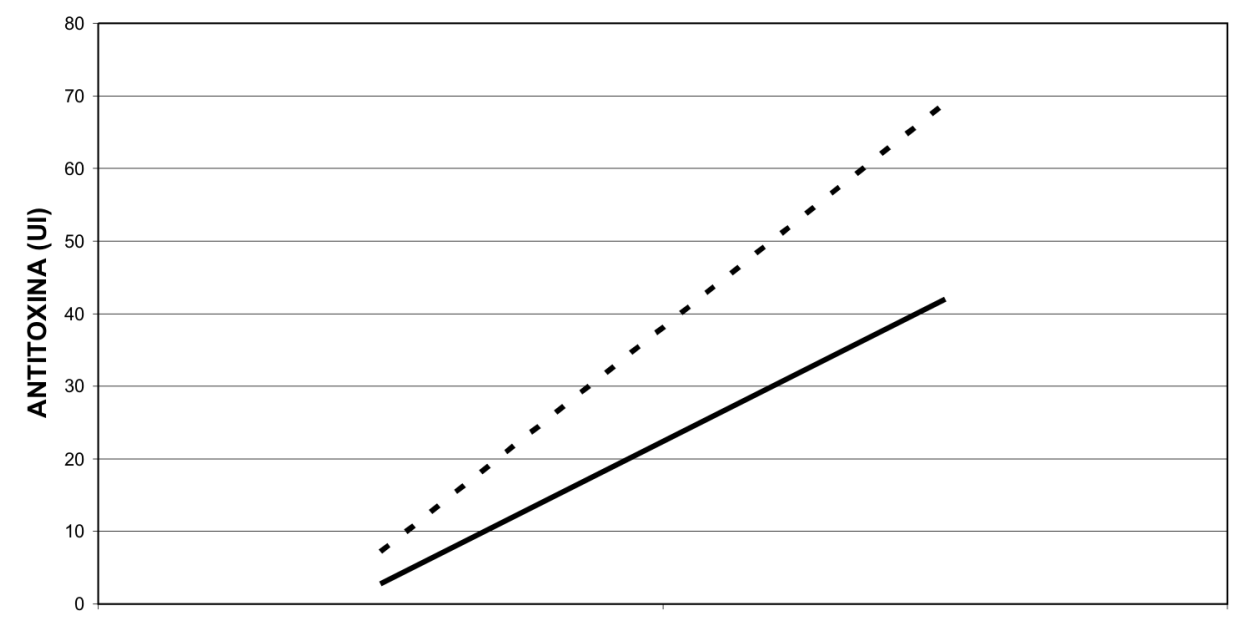

Gráfica 2. Comparación entre lotes del toxoide tetánico (x) Lote 11, (y) Lote 69 y adyuvante 1 (--------) y adyuvante 2 (

fuerte, tenga como resultado una lenta liberación del antígeno y, por ende, una mejor presentación del antígeno al sistema inmune, esto podría explicar la mejor efectividad del adyuvante.

Aggerbeck et al. (1996) realizaron un estudio donde comparan estos dos adyuvantes en pruebas de potencia in vivo y determinaron que el hidróxido de aluminio era mas efectivo que el fosfato de calcio. Sin embargo, un estudio posterior (Aggerbeck et al. 1997) realizado al nivel clínico con soldados, demostró todo lo contrario, que el fosfato de calcio genera mejores respuestas inmunes pero mayores efectos adversos. En conclusión, según los datos del análisis estadístico entre los dos adyuvantes no hay demasiada diferencia como si se observa con la variable lote. Sin embargo, y según la variabilidad de los resultados no solo de este estudio sino de los anteriormente referenciados demuestra que la reacción biológica a los diferentes adyuvantes varía significativamente. 
Los datos arrojados por la variable concentración frente a la respuesta inmunogénica muestran una relación aparentemente lineal entre la concentración formulada y la repuesta inmunogénica, es decir, a mayor concentración de toxoide mayor respuesta inmunogénica. La importancia de estos datos, se encuentra en que se demuestra que independientemente de los lotes o los adyuvantes utilizados sí se puede bajar la concentración de toxoide en la formulación y, a su vez, garantizar que la producción de antitoxina sea de un nivel de $2 \mathrm{UI} / \mathrm{mL}$, lo que confirma que se está desperdiciando toxoide en la formulación.

Al comparar la concentración de toxina y el lote frente a la respuesta inmunogénica, se obtienen conclusiones interesantes, como que el lote 11 en las tres concentraciones presenta títulos menores que el lote 69. Esto contradice el rumbo de las investigaciones sobre el mejoramiento de producción y de rendimiento de la toxina antitetánica, pues se ha verificado que el cultivo agitado es superior en rendimiento, en volumen final y en tiempo de producción al cultivo estático, además que reduce la manipulación del producto (Arias et al. 2003).

En cuanto a los resultados obtenidos de la variable lote frente a la respuesta inmunogénica, se evidencia que el lote 69 es mejor que el lote 11. En definitiva, la diferencia en el método de producción de los lotes de toxoide tetánico demuestra que éste afecta directamente la respuesta inmunogénica. Al comparar las dos técnicas de producción, el medio de cultivo utilizado, la cepa, la concentración de inoculo, el pH y la temperatura son iguales y lo único que varia es la condición de agitación y el tiempo de producción, lo que llevaría a pensar que el oxígeno afecta directamente la calidad de la toxina y respecto al adyuvante, se concluye que el $\mathrm{Al}(\mathrm{OH})_{3}$ presenta mejores resultados en cuanto a la efectividad de la vacuna que el $\mathrm{Ca}_{3}\left(\mathrm{PO}_{4}\right)_{2}$.

Las vacunas inactivadas requieren agonistas inmunes (inmunopotenciadores o adyuvantes) para determinar el tipo de inmunidad inducida (Th1 o Th2) y promover la respuesta inmunológica. Debido a que los agonistas, además de afectar la inmunidad, también pueden modificar la producción de linfocitos T citotóxicos (CTL), su selección es crítica para la efectividad de las vacunas. Aunque la síntesis de CTL constituye un objetivo para las vacunas, en donde la muerte de las células blanco por parte de los CTL es una meta en sí misma, en otras vacunas esto se complica en virtud de las exacerbaciones potenciales que pueden promover sobre enfermedades como la hepatitis y la de Alzheimer, en donde la producción de CTL, se debe evitar. El descubrimiento de que los agonistas, al actuar sobre diferentes receptores, puede aumentar sinérgicamente la inmunidad, ha abierto nuevas posibilidades para la síntesis de nuevas vacunas. El hecho de que algunos polisacáridos con modificaciones específicas, potencian la inmunidad Th1, contribuirá al descubrimiento de compuestos sin autoinmunidad asociada. La comprensión de los mecanismos inmunomoduladores de los agonistas también sustentará la utilización de estos agentes como tratamientos independientes (Marciani, 2007)

Las propiedades adyuvantes de las sales de aluminio presentan limitaciones; actualmente se está evaluando una amplia gama de adyuvantes nuevos para su uso en vacunas nuevas o mejoradas. Estas investigaciones están siendo lideradas por el comité consultivo mundial sobre seguridad de vacunas de la OMS (2006). Son ejemplos de estos adyuvantes los estimuladores del sistema inmunitario, los vehículos formados por micropartículas y las emulsiones, así como diversas combinaciones de éstos. Se espera que los adyuvantes permitan el desarrollo de vacunas inocuas y eficaces contra enfermedades para las que aún no hay vacunas, como la malaria y el $\mathrm{VIH}$, así como mejorar la eficacia de otras vacunas. Dado que muchos de los adyuvantes nuevos se utilizarán probablemente en vacunas para enfermedades endémicas en países en desarrollo, será preciso disponer de sistemas para vigilar la seguridad de estas vacunas nuevas (OMS, 2006).

Para analizar la seguridad de los nuevos adyuvantes, una vez combinados en una vacuna, se necesitan estándares mejorados y coherentes que rijan las juntas de seguimiento de la seguridad, la generación de señales y la evaluación posterior para la autorización, la comercialización de las vacunas y la vigilancia de los eventos inmunológicos adversos e inclusive problemas de seguridad inesperados. Los estudios de ultima fase deben abordar las reacciones adversas que no pueden ser previstas a través de los efectos biológicos de la vacuna y del adyuvante, incluidas las reacciones adversas raras e infrecuentes que no se detectan en los estudios con animales o en los estudios con pocas personas realizados antes de la homologación de la vacuna. Para ello, se necesitan definiciones de caso claras. Todo ello 
exige un enfoque clínico, científico y regulador nuevo, que tenga en cuenta la seguridad de los adyuvantes a corto y largo plazo (Vax, 2006).

\section{BIBLIOGRAFÍA}

AGGERBECK, H.; FENGER, C.; HERON, I. 1995. Booster vacination against Diphteria and Tetanus in man. Comparison of calcium posphate and aluminium hidroxide as adyuvants - II. Vaccine. 13(14):1366-1374.

AGGERBECK, H.; WANTZIN, J.; HERON, I. 1996. Booster vacination against Diphteria and Tetanus in man. Comparison of three different vaccine formulations - III. Vaccine. 14(13):1265-1272.

AGGERBECK, H.; GIZURARSON, S.; WANTZIN, J.; HERON, I. 1997. Intranasal booster vaccination against diphtheria and tetanus in man. Vaccine. 15;307-316.

ARIAS, J.; CÉSPEDES, M.; DÍAZ, S.; GUTIÉRREZ, I. 2003. Evaluación de la influencia de la agitación y de la concentración de formaldehído en la detoxificación de la toxina tetánica. Universitas Scient. 8(2):23-30.

AZUMA, I. 1992. Synthetic immunoadjuvants: application to nonspecific host stimulation and potentiation of vaccine immunogenicity. Vaccine. 10:10001006.

BARRETO, A. R. 2000. Estudio preliminar de la fermentación de Clostridium tetani, por cultivo continuo. Tesis de maestría. Facultad de Ingeniería. Universidad Nacional de Colombia. p.250.

BENNET, B.; CHECK, I.J.; OLSEN, M.R.; HUNTER, R.L. 1992. A comparison of commercially available adjuvants for use in research. J. Immunol. Methods 153:31-40.

DILUZIO, N.R. 1985. Update on the immunomodulating activities of glucans. Springer Semin. Immunopathol 8:387-400.
GUERRERO, J.; GATTAS, C.R. 1982. Immunolomodulating substances: an overview. Rev Microbiol. 13(2):110115.

GUPTA, R.; RELYVELD, E. 1991. Adverse reactions after injection of adsorbed Diphteria -PertussisTetanus (DPT) vaccine are not due only to Pertussis organisms or Pertussis components in the vaccine. Vaccine. 9:669-702

INSTITUTO NACIONAL DE SALUD (INS). 1995. Manual de procedimientos del laboratorio de Tetanos. Bogotá. 120p.

INSTITUTO NACIONAL DE SALUD (INS). 1998. Instituto Nacional De Salud. 80 años. Una historia, Un Compromiso. Bogotá (Colombia). 140p.

LATHAM, W.C.; BENT, D.F.; LEVINE, L. 1962. Tetanus toxin production in the abscense of protein. Appl. Microbiol 10:142-146.

MARCIANI DANTE, J. 2007. Avantogen. Ed. SIIC 2a Ed. 22p.

ORGANIZACIÓN MUNDIAL DE LA SALUD (OMS). 1997. Vacunas e Inmunización. Situación actual. Ginebra. 25p.

ORGANIZACIÓN MUNDIAL DE LA SALUD (OMS). 2006. Vacunas e Inmunización: Situación actual. Ginebra. 10p.

REYNOLDS, J.A.; KASTELLO, M.D.; HERRIGTON, D.G.; CRABBS, C.L.; PETERS, C.J.; JEMSKI, J.V. 1980. Glucan-induced enhancement of host resistance to selected infectious diseases. Infect. Immun. 30:51-57.

ROITT, I.M. 1991. Essential Immunology. 7 ed. Oxford: Blackwell Scientific, 326p.

SPRIGGS, D.; KOFF, W. 1991. Topics in Vaccine Adjuvant Research. CRC Press. United States. 145p.

STEWART-TULL, D.E.S. 1989. Recommendations for the assessment of adjuvants (immunopotentiators). En: Gregoriadeis, G.; Allison, A.C.; Poste, G, eds. 
Immunological adjuvants and vaccines. New York: Plenum, p.213-226.

TAKX-KOHLEN, B.C. 1992. Immunomodulators. Future prospects. Pharm. Weekbl. Science 14:24-52.

UNITED STATES HEALTH AND HUMAN SERVICES. 2003. Division of immunization, Atlanta, Georgia. Surveillance for Safety After Immunization: Vaccine Adverse Event Reporting System published on January 24, 2003 Vol. 52, No. SS-1.
VAX. 2006. Nature's adjuvant. IAVI Report Bulletin 4(10), Octubre 2006. Disponible desde Internet en: http://www.iavireport.org/vax/VAXOctober2006. asp (con acceso 05/03/07).

WAGNEROVÁ, J.; FERENCÍK, M. 1993. Secretory and regulatory products of macrophagues in the immune and inflamatory reactions. Biol. Bratislav. 48(6):709-717.

Recibido: Junio 23 de 2005

Aceptado: Marzo 26 de 2007 